\title{
Discrete foraging niches promote ecological, phenotypic, and genetic divergence in sympatric whitefish (Coregonus lavaretus)
}

\author{
Anna Siwertsson • Rune Knudsen • Kim Præbel • Colin E. Adams • \\ Jason Newton $\cdot$ Per-Arne Amundsen
}

Received: 5 April 2012/ Accepted: 7 September 2012/Published online: 23 September 2012

(C) The Author(s) 2012. This article is published with open access at Springerlink.com

\begin{abstract}
Natural populations often vary in their degree of ecological, morphological and genetic divergence. This variation can be arranged along an ecological speciation continuum of increasingly discrete variation, with high inter-individual variation at one end and well defined species in the other. In postglacial fishes, evolutionary divergence has commonly resulted in the co-occurrence of a pelagic and a benthic specialist. We studied three replicate lakes supporting sympatric pelagic and benthic European whitefish (Coregonus lavaretus (L.)) morphs in search for early signs of possible further divergence into more specialized niches. Using stomach content data (recent diet) and stable isotope analyses (time-integrated measure of trophic niche use), we observed a split in the trophic niche within the benthic whitefish morph, with individuals specializing on either littoral or profundal resources. This divergence in resource use was accompanied by small but significant differences in an adaptive morphological trait (gill raker number) and significant genetic differences between fish exploiting littoral and profundal habitats and foraging resources. The same pattern of parallel divergence was found in all three lakes, suggesting similar natural selection pressures driving and/or maintaining the divergence. The two levels of divergence (a clear and robust benthic - pelagic and a more subtle littoral profundal divergence) observed in this study apparently represent different stages in the process of ecological speciation.
\end{abstract}

Electronic supplementary material The online version of this article (doi:10.1007/s10682-012-9607-x) contains supplementary material, which is available to authorized users.

A. Siwertsson $(\bowtie) \cdot$ R. Knudsen $\cdot$ K. Præbel $\cdot$ P.-A. Amundsen

Department of Arctic and Marine Biology, University of Troms $\varnothing, 9037$ Troms $\emptyset$, Norway

e-mail: anna.siwertsson@uit.no

C. E. Adams

Scottish Centre for Ecology and the Natural Environment, University of Glasgow, Rowardennan, Glasgow G63 0AW, Scotland, UK

J. Newton

NERC Life Sciences Mass Spectrometry Facility, SUERC, East Kilbride G75 0QF, Scotland, UK 
Keywords Resource polymorphism - Adaptive radiation - Parallel evolution ·

Trophic specialization $\cdot$ Natural selection $\cdot$ Stable isotopes

\section{Introduction}

The process of adaptive radiation and ecological speciation may generate a continuous pattern of increasingly discrete variation, with high inter-individual variation at one end through discrete polymorphism, to well defined separate species in the other (Seehausen et al. 2008b; Hendry et al. 2009; Peccoud et al. 2009; Seehausen 2009; Nosil 2012). The concepts of adaptive radiation and ecological speciation similarly state that adaptations to local environments and barriers to gene flow evolve between populations as a result of ecologically-based divergent selection (Schluter 2000; Rundle and Nosil 2005). Intraspecific competition is one source of such divergent selection, and small-scale differences between individuals in resource use have been recognized as an important initial step towards further population divergence (Bolnick et al. 2003; Hendry 2009; Hendry et al. 2009). In vertebrate adaptive radiations, inter-individual variation and divergence in habitat use and/or diet choice is common in the early stage of population divergence (Streelman and Danley 2003; Räsänen and Hendry 2008; Puebla 2009). When observed differences in resource use are stable over time, morphological adaptations and polymorphisms may evolve (Skúlason and Smith 1995; Skúlason et al. 1999; Streelman and Danley 2003; Puebla 2009). Divergent natural selection between environments may reduce gene flow between ecotypes through direct selection for assortative mating (reinforcement; Servedio and Noor 2003), or through the evolution of any kind of reproductive barrier as a by-product of ecological divergence (e.g. Coyne and Orr 2004; Nosil 2012). Ultimately, the process may lead to the formation of separate species, i.e. ecological speciation (Schluter 2001; Rundle and Nosil 2005).

Some of the most spectacular radiations of vertebrates are documented in fishes in freshwater lakes, e.g. the species flocks of cichlids in East African lakes (Kocher 2004) and diversity of sailfin silversides in the Malili Lakes (Herder and Schliewen 2010). Many examples of recent (10-15,000 years) adaptive radiation come from fishes in postglacial freshwater systems, which typically manifests as the co-occurrence of a pelagic and a benthic specialized morph or species (Schluter and McPhail 1993; Robinson and Wilson 1994; Skúlason and Smith 1995). Aquatic ecosystems typically offer several, often discrete, foraging resources for fish. In addition to divergence into pelagic and benthic niches, in some temperate fish species further divergence into more specialized niches has been documented e.g. into profundal (Kahilainen et al. 2004; Knudsen et al. 2006), and preyspecific benthic niches (Knudsen et al. 2011), or piscivory (Malmquist et al. 1992; Adams et al. 1998), and in some cases to more system specific niches such as the lava structures in Icelandic lakes (Snorrason et al. 1994; Kristjánsson et al. 2002). Here we examine the early phase of a possible divergence beyond the classical pelagic-benthic dichotomy, in European whitefish (Coregonus lavaretus (L.)) in a sub-arctic watercourse.

European whitefish is common in postglacial lakes in northern Fennoscandia, where two discrete morphs adapted to utilizing pelagic and benthic resources commonly co-occur (Østbye et al. 2006; Siwertsson et al. 2010). The two morphs differ in ecology and in morphological traits related to resource exploitation, particularly in the number of gill rakers (Amundsen et al. 2004a; Siwertsson et al. 2010; Kahilainen et al. 2011). The pelagic morph (referred to as the densely rakered morph, DR; Kahilainen et al. 2004) has many, 
long, densely packed gill rakers, and is a zooplanktivore specialist (Amundsen et al. 2004a, b; Kahilainen et al. 2011). The benthic morph (referred to as the large sparsely rakered morph, LSR; Kahilainen et al. 2004) is a more general forager feeding on both benthic prey and zooplankton, and has shorter, fewer and more widely spaced gill rakers (Knudsen et al. 2003; Amundsen et al. 2004a, b; Kahilainen and Østbye 2006). A third whitefish morph (small sparsely rakered, SSR), specializing on profundal resources, is known to coexist with the former two in some lakes in the Paatsjoki/Pasvik watercourse in the border region between Finland, Norway and Russia (Kahilainen et al. 2004, 2011), but is unrecorded and apparently absent from other lake systems in northern Fennoscandia (Amundsen et al. 2004b; Siwertsson et al. 2010). This is surprising given the availability of profundal foraging resources in many postglacial lakes and the existence of a specialized profundal feeding whitefish morph elsewhere (Kahilainen et al. 2004; Siwertsson et al. 2010).

In this study we test if the discrete nature of the pelagic, littoral, and profundal foraging resources is supporting a common pattern of ecological, phenotypic, and genetic structuring of whitefish populations indicating parallel patterns of divergence. First, we hypothesized that all three discrete foraging strategies (pelagic, littoral, profundal) have promoted an ecological and morphological specialism amongst whitefish from three sites where profundal specialists have not been recorded. Second, that the resource specialist groups are reproductively isolated. Third, that resource specialists show parallel patterns of specialism across three contrasting lakes.

\section{Materials and Methods}

Study area and sampling

The present study was conducted in three lakes known to support densely (DR) and large sparsely rakered (LSR) whitefish (Siwertsson et al. 2010). The lakes Lahpojavri (LP) $\left(69.25^{\circ} \mathrm{N}, 23.78^{\circ} \mathrm{E}\right)$, Suopatjavri (SU) $\left(68.93^{\circ} \mathrm{N}, 23.09^{\circ} \mathrm{E}\right)$ and Vuolgamasjavri (VG) $\left(69.14^{\circ} \mathrm{N}, 23.36^{\circ} \mathrm{E}\right)$ are all situated in the Alta-Kautokeino watercourse in the sub-arctic region of northern Norway. Lahpojavri and Suopatjavri are isolated from the other lakes based on water-flow direction and the presence of waterfalls and rapids, whilst it is possible that fish might move downstream from Suopatjavri to Vuolgamasjavri, migration rate (if it occurs) is not known. The lakes are oligotrophic with some humic impact from the surrounding tundra (Siwertsson et al. 2010). They are of varying size, but all have welldeveloped pelagic, littoral (shallow benthic habitats with $>1 \%$ of surface light levels) and profundal (deep benthic habitats with $<1 \%$ of light at surface) zones (Table 1). We use the term "benthic" to collectively refer to littoral and profundal environments. Whitefish dominate the fish community in all three lakes, but perch (Perca fluviatilis L.), pike (Esox lucius L.), burbot (Lota lota (L.)), brown trout (Salmo trutta L.), Arctic charr (Salvelinus alpinus (L.)), and minnow (Phoxinus phoxinus (L.)) are also present.

Fish and their putative prey were sampled from all three principal lake habitats, pelagic (0-6 m), littoral (1-8 m), and profundal (18-35 m), during late August - early September in 2007 or 2008. Fish were collected using multi-mesh $(10-45 \mathrm{~mm})$ survey gillnets set overnight. The whitefish were assigned to morph in the field by evaluation of appearance, head and body form and a visual evaluation of the gill raker morphology (Amundsen 1988; Amundsen et al. 2004a; Kahilainen and Østbye 2006; Harrod et al. 2010). The DR whitefish are usually of small size, silvery and have long, thin, and densely packed gill rakers. The LSR whitefish are larger in size with typical whitefish coloration with silvery 
Table 1 Physical characteristics of the three study lakes

\begin{tabular}{llllllll}
\hline Lake & $\begin{array}{l}\text { Area } \\
\left(\mathrm{km}^{2}\right)\end{array}$ & $\begin{array}{l}\text { Perimeter } \\
(\mathrm{km})\end{array}$ & $\begin{array}{l}\text { Max depth } \\
(\mathrm{m})\end{array}$ & $\begin{array}{l}\text { Mean depth } \\
(\mathrm{m})\end{array}$ & $\begin{array}{l}\text { Pelagic } \\
(\%)\end{array}$ & $\begin{array}{l}\text { Littoral } \\
(\%)\end{array}$ & $\begin{array}{l}\text { Profundal } \\
(\%)\end{array}$ \\
\hline Lahpojavri & 8.1 & 46.3 & 36 & 8.7 & 42 & 58 & 42 \\
Suopatjavri & 2.0 & 10.5 & 25 & 8.2 & 39 & 61 & 39 \\
Vuolgamasjavri & 1.2 & 19.7 & 30 & 14.9 & 73 & 27 & 73 \\
\hline
\end{tabular}

Availability of pelagic, littoral and profundal habitats is measured in percent of lake surface area

sides, dark back and fins, and shorter, robust gill rakers with wider spacing. No individuals with typical SSR whitefish appearance, i.e. large eyes and head, subterminal mouth, reddish fins, and extremely short and widely spaced gill rakers, were detected in the present study lakes. Throughout this paper, we use the terms DR and LSR whitefish morphs to refer to this a priori classification. Fish were measured (fork length) and a sample of muscle tissue was taken just below the dorsal fin for stable isotope analysis from about 25 randomly selected individuals from each habitat. The left first branchial arch of the gill was dissected, and the number of gill rakers counted. Stomach fullness was visually determined on a percentage scale from empty $(0 \%)$ to full $(100 \%)$. Prey items were determined to lowest feasible taxonomic level and their contribution to the total fullness was estimated (Amundsen 1995). Prey were categorized into nine groups and divided into habitat specific groupings based on dominant occurrence: Pelagic prey-cladocerans, copepods, and large pelagic prey (surface insects and insect pupae); littoral prey-Eurycercus lamellatus, large crustaceans (Gammarus lacustris and Asellus aquaticus), insect larvae, and snails; profundal prey-mussels (mainly Pisidium spp.), and chironomid larvae. Only fish larger than $10 \mathrm{~cm}$ were included in this study, to reduce effects of ontogenetic niche shifts (Sandlund et al. 1992). Sample sizes used in comparisons of different variables between groups of whitefish are given in Table S1 (electronic supplementary). For analysis of stable isotope ratios, benthic prey were collected by an Ekman grab from the profundal and by grab, pond net, and kick sampling in littoral areas. Benthic prey were sorted into the same categories as used for stomach content analyses. Zooplankton bulk samples were collected by plankton net in pelagic habitats over the deepest area in each lake.

\section{Stable isotope measurements}

Stable isotope ratios of carbon $\left(\delta^{13} \mathrm{C}\right)$ and nitrogen $\left(\delta^{15} \mathrm{~N}\right)$ are important as a complement to traditional stomach content analyses because they give a longer-term integrated signal of food intake as opposed to the "snapshot" information from stomach contents. Stable isotope ratios from fish muscle tissue typically reflect assimilated food during the summer growth period (Perga and Gerdeaux 2005). Information about the temporal consistency of diets is especially important for assessing individual specialization (Bolnick et al. 2002), and stable isotopes are now also being used in studies of ecological niche (e.g. Layman et al. 2007a; Newsome et al. 2007; Quevedo et al. 2009). With traditional stomach content analyses it may also sometimes be difficult to distinguish between littoral and profundal foraging niche use because some prey groups/species (e.g. chironomid larvae and Pisidium spp.) may occur in both habitats. Stable isotopes can distinguish between resources from the three principal habitats in lakes (Vander Zanden and Rasmussen 1999; Syväranta et al. 2006; Harrod et al. 2010). Littoral and pelagic resources typically differ in baseline isotopic values since pelagic phytoplankton are depleted in $\delta^{13} \mathrm{C}$ compared to benthic algae 
(Vander Zanden and Rasmussen 1999). Profundal areas are often dominated by the detritus food chain, which gives more enriched $\delta^{15} \mathrm{~N}$ due to the accumulation of the heavier isotope in consumers compared to their prey (e.g. Vander Zanden and Rasmussen 1999; Post 2002). Generally $\delta^{13} \mathrm{C}$ increases by 0.4 for and $\delta^{15} \mathrm{~N}$ by 3.4 for each trophic level (Post 2002).

Samples of fish muscle tissue and invertebrate prey for analyses of stable isotopes were dried at $60{ }^{\circ} \mathrm{C}$, then ground to a fine powder with a mortar and pestle. Invertebrates were analyzed as bulk samples of whole organisms since individuals of most prey groups were too small for dissection and removal of soft tissue. Carbonate rich invertebrate samples (crustaceans and molluscs) were divided into two sub-samples: one was left untreated for $\delta^{15} \mathrm{~N}$ measurements, while the second was acidified to remove any inorganic carbon before analysis for $\delta^{13} \mathrm{C}$. Acidification was performed by adding $10 \% \mathrm{HCl}$ drop by drop until no further $\mathrm{CO}_{2}$ gas bubbles were observed (Kang et al. 2003; Jacob et al. 2005). The samples were rinsed with distilled water until $\mathrm{pH} 6$ was attained in the sample, centrifuged (4000 rpm, $5 \mathrm{~min}$ ) and the supernatant removed each time, before being finally dried $\left(60{ }^{\circ} \mathrm{C}, 24 \mathrm{~h}\right)$. Analysis of carbon $\left(\delta^{13} \mathrm{C}\right)$ and nitrogen $\left(\delta^{15} \mathrm{~N}\right)$ stable isotope ratios were performed at the NERC Life Sciences Mass Spectrometry Facility, by continuous flow isotope ratio mass spectrometry (CF-IRMS), using a Costech ECS 4010 elemental analyser coupled to a ThermoFisher Scientific Delta XP-Plus IRMS. Stable isotope values are given in per mil (\%) in the conventional delta format in relation to the international standards Vienna PeeDee Belemnite for $\delta^{13} \mathrm{C}$ and atmospheric nitrogen for $\delta^{15} \mathrm{~N}$. Low values of C:N ratios in the analyzed fish samples (C:N mean: 3.18, range: 3.01-3.39) indicated no need for lipid correction (Kiljunen et al. 2006; Post et al. 2007).

Genetic analyses

Genomic DNA was extracted from gill filaments using E-Z96 Tissue DNA Kit (OMEGA Bio-tek) following the manufacturer's instructions. A total of 16 neutral microsatellite loci (Table S2) were amplified using forward-primer labeled primers in four PCR multiplexes following the protocol by Præbel et al. (in press). The PCR products were separated on an ABI 3130 XL Automated Genetic Analyzer (Applied Biosystems) using GENESCAN LIZ500 (Applied Biosystems) size standard. The binning and scoring was performed in GENEMAPPER 3.7 (Applied Biosystems) as described in Præbel et al. (in press). Replicate $(5 \%)$ and blind (4\%) samples were included in all analysis to confirm consistency of scoring and absence of contamination. The samples were screened for abnormalities in the software MICRO-CHECKER 2.2.3 (Van Oosterhout et al. 2004), using 1000 bootstraps to generate the expected homozygote and heterozygote allele size difference frequencies.

Standard genetic diversity measures, $\mathrm{Na}, \mathrm{H}_{\mathrm{e}}, \mathrm{H}_{\mathrm{o}}$, and $\mathrm{F}_{\mathrm{IS}}$, as well as deviations from Hardy-Weinberg equilibrium (HWE) and linkage equilibrium (LE) were estimated using GENEPOP 4.0 (Rousset 2008). Departures from HWE and LE were tested by exact tests (Guo and Thompson 1992) and the number of significant comparisons before and after Sequential Bonferroni corrections (Rice 1989) for multiple comparisons are presented. These standard genetic measures are presented in electronic supplementary (Table S3). We also tested for departures from HWE and LE in pooled samples of littoral and profundal caught LSR to reveal any signatures of the Wahlund effect. We tested whether a priori defined groups could be genetically discriminated by pair-wise $\mathrm{F}_{\mathrm{ST}}$ (Weir and Cockerham 1984), using ARLEQUIN 3.5.1.2 (Excoffier and Lischer 2010) with Sequential Bonferroni corrections for multiple comparisons. We also tested for genetic sub-groups within LSR whitefish using the Bayesian clustering method of STRUCTURE 2.3.2 (Pritchard et al. 
2000; Hubisz et al. 2009), under a model assuming admixture and correlated allele frequencies between $\mathrm{k}$ population groups (Burn-ins of 1,000,000 replications and 1,500,000-2,000,000 Markov chain Monte Carlo (MCMC) replicates). We used habitats (littoral and profundal) as prior information to assist the structuring (the LOCPRIOR model) as recommended for weak signals of structuring (Hubisz et al. 2009). All runs were replicated 15 times at $\mathrm{K}=1-3$ to confirm consistency of log-likelihood probabilities. The most likely (highest $\ln \operatorname{Pr}(X \mid K)$ ) grouping was visualized using STRUCTURE HARVESTER (Earl and vonHoldt 2011).

Statistical analyses

Habitat distribution (\%) for each morph was estimated based on catch per unit effort (CPUE; defined as the number of fish caught per $100 \mathrm{~m}^{2}$ gill net per night) in pelagic, littoral and profundal habitats. Niche overlap in habitat and diet based on stomach content was determined using Schoeners index (Schoener 1970). This index ranges from 0 (complete niche segregation) to 1 (complete niche overlap). Following Wallace (1981) an overlap $\geq 0.6$ is interpreted as biologically significant. Diet niche width for each group was calculated from stomach contents using the Levins index, B (Levins 1968). In this study B ranges from 1 (one prey category) to 9 (equal representation of all prey categories). The degree of individual diet specialization within populations and the extent to which diet variation is arranged in discrete groups were quantified using the program DIETA1 (Araujo et al. 2008). The index of individual diet specialization (E) measures the mean pairwise diet overlap between all individuals in a population and ranges from 0 when there is no inter-individual diet variation to 1 as the diet variation is increasing (Araujo et al. 2008). The clustering index (C) measures the degree to which individuals in a population are organized into discrete clusters with little diet overlap with other groups, where a value of 0 represents no clustering, positive values towards +1 represents clustered populations and negative values $(-1)$ are the result of overdispersed individual diets (Araujo et al. 2008).

Isotopic niche measures were calculated using the methods of Layman et al. (2007a) and Jackson et al. (2011) based on values of $\delta^{13} \mathrm{C}$ and $\delta^{15} \mathrm{~N}$. Layman et al. (2007a) suggested several community measures based on the use of stable isotopes, which has successfully been applied also at the level of populations (e.g. Layman et al. 2007b; Quevedo et al. 2009). The niche width of each group was described by the area the population occupies on a $\delta^{13} \mathrm{C}-\delta^{15} \mathrm{~N}$ biplot. The area was determined by a Bayesian estimate of the standard ellipse area (SEA; similar to standard deviation but for bivariate data) as described in Jackson et al. (2011), and implemented in the package siar (version 4.1.1) for R (Parnell et al. 2010). Probability values for differences between groups were obtained by calculating the proportion of the total number of simulations $(10,000)$ where one group had a larger SEA than the other. Isotopic niche variability (average degree of trophic diversity) within groups was calculated as the mean Euclidean distance of individuals to the centroid (CD). The centroid is the mean $\delta^{13} \mathrm{C}$ and $\delta^{15} \mathrm{~N}$ values of all the individuals within the group, and describes the isotopic niche position. Statistical tests of differences in centroid location and $\mathrm{CD}$ were performed with a residual permutation procedure (RPP) as described in Turner et al. (2010). The absolute value of the difference of metrics between samples was used as a test statistic, and was considered significant if greater than zero. Test statistics were compared to null distributions obtained from 9999 permutations of residuals from reduced linear models via the RPP procedure (Turner et al. 2010). We also used the parametric Hotelling's $T^{2}$ test statistic, a multivariate analogue to the $t$ test. 
To further explore the occurrence of ecological sub-groups based on values of stable isotope ratios, we used a model-based clustering approach implemented in the package mclust (version 3) for R (Fraley and Raftery 2006). With this method, the observed frequency distributions of $\delta^{13} \mathrm{C}$ and $\delta^{15} \mathrm{~N}$ were fitted to a number of alternative models with one up to a mixture of six Gaussian distributions. A similar approach was used to objectively examine modality in gill raker number distributions. With univariate data, observations are fitted to models with one or a mixture of up to three Gaussian distributions. The best model, and the estimated number of clusters, was selected based on the Bayesian Information Criterion (BIC, analogous to Akaike's Information Criterion; Fraley and Raftery 2002). For each population we compared the best model with the next best model (resulting in a different number of groups) by calculating $\triangle \mathrm{BIC}$ as the difference in the BIC-values between the best model and the next best model. Following Kass and Raftery (1995) we interpreted $\triangle \mathrm{BIC}>10$ as very strong support for the best model, $6<\Delta \mathrm{BIC}<10$ as strong support, $2<\Delta \mathrm{BIC}<6$ as moderate support, and $\Delta \mathrm{BIC}<2$ as equivalent support for the best and the next best model. Statistical analyses were conducted in the R statistical computing package (R Development Core Team, 2011).

\section{Results}

Pelagic and benthic foraging specialisms

Gill raker frequency distributions had a bimodal pattern in all three lakes (Fig. 1a), corresponding to and confirming the a priori assignment of fish to DR and LSR morphs using external characters. The DR morph had a significantly higher number of gill rakers than the LSR morph in all lakes ( $t$ tests all lakes: $p<0.001$ ) (Fig. 1a). However, there were slight differences in absolute number of gill rakers of the two morphs between lakes (ANOVA DR: $\mathrm{F}_{2,139}=12.5, p<0.001$; LSR: $\mathrm{F}_{2,273}=29.6, p<0.001$ ). Post hoc Tukey's HSD tests showed that the LSR whitefish in Vuolgamasjavri had significantly lower number of gill rakers than in the other two lakes $(p<0.001)$, while DR whitefish in Suopatjavri had significantly higher numbers than in other lakes $(p<0.001)$. There was also a slight difference in gill raker numbers between LSR whitefish from Lahpojavri and Suopatjavri $(p=0.032)$. The relative abundance of the two morphs was similar in Suopatjavri (54\% DR and $46 \%$ LSR), while LSR whitefish was the more abundant in Vuolgamasjavri (88 \%), and DR whitefish the more abundant in Lahpojavri (88 \%).

The two morphs were separated in niche use, as indicated by low overlap in both habitat (Fig. 1b) and diet (Fig. 2) in all three lakes (Schoeners index means between morphs within lakes: habitat: 0.31 , diet: 0.30 , Table S4, S5). LSR whitefish almost exclusively used benthic (littoral and profundal) habitats and prey, while the DR whitefish fed almost solely on planktonic prey and was primarily recorded in the pelagic habitat (Fig. 1b, 2). The diet niche width of DR was considerably smaller (mean $\mathrm{B}=1.86$, Table S6) than for the LSR morph (mean B = 4.80), and the degree of inter-individual diet variation was also lower in the DR morph (DR: mean $\mathrm{E}=0.38$, LSR: $\mathrm{E}=0.74$, Table S6). Based on stomach contents, diet variation was not organized into clusters in either morph (Table S6). DR and LSR whitefish morphs utilized similar habitats and prey resources in the different lakes, which was evident by high niche similarity indices within morphs between lakes (Schoeners index means (LSR; DR): habitat: 0.88; 0.72, diet: 0.59; 0.48, Table S4, S5).

Isotopic values from prey in the three principal lake habitats are presented in Table S7. In general, prey from littoral habitats were the most enriched in $\delta^{13} \mathrm{C}$, while profundal prey 
(a)
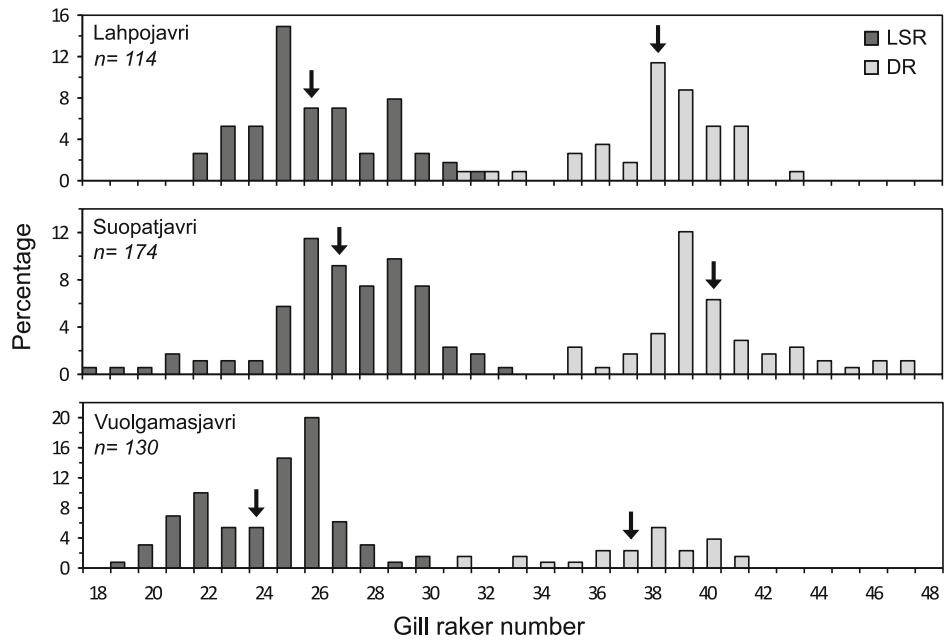

(b)

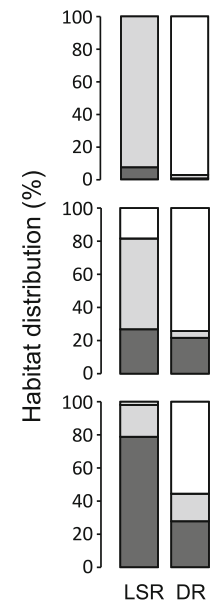

Fig. 1 a Gill raker frequency distributions for the large sparsely rakered (LSR) and the densely rakered (DR) whitefish morphs. Arrows indicate mean gill raker number for each morph. b Habitat distribution (\%) of the LSR and DR morphs based on CPUE. White depicts pelagic, grey littoral, and black profundal habitat

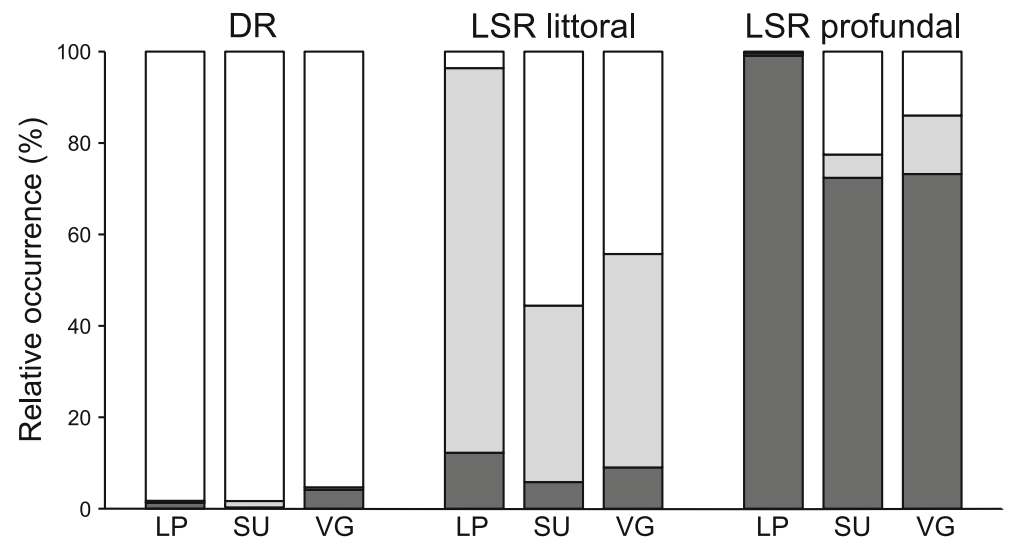

Fig. 2 Diet choice based on prey occurrence in stomach contents of densely rakered (DR) and large sparsely rakered (LSR) whitefish collected from the littoral and the profundal habitat in Lahpojavri (LP), Suopatjavri (SU), and Vuolgamasjavri (VG). White depicts pelagic, grey littoral, and black profundal prey

were more enriched in $\delta^{15} \mathrm{~N}$ compared to littoral prey. Profundal and pelagic prey organisms had more similar isotopic composition.

The two morphs had significantly different isotopic niches (centroid location) in Lahpojavri (permutation test: $p=0.001$, Hotelling's $\mathrm{T}^{2}: p<0.001$ ) and Vuolgamasjavri (permutation test: $p=0.007$, Hotelling's $\mathrm{T}^{2}: p<0.001$ ), but not in Suopatjavri (permutation test: $p=0.088$, Hotelling's $\mathrm{T}^{2}: p=0.20$; Fig. 3). The isotopic niche was on average 11 times larger in the LSR compared to the DR morph (SEA difference LSR-DR: all lakes 
$p<0.001$; Table S6). The variability in niche use was also significantly higher in the LSR morph (CD permutation test: LP, VG: $p=0.001$, SU: $p=0.002$, Table S6).

Next, we wanted to identify possible foraging specialist groups within each morph based on model-based clustering using the stable isotope ratios. Supporting the results of low inter-individual variation in diet use within the DR whitefish, no clear ecological subgroups were found within this morph in Lahpojavri or Suopatjavri ( $\triangle \mathrm{BIC}$ : 1.5 and 1.0). In Vuolgamasjavri, two clusters were identified within the DR whitefish ( $\triangle \mathrm{BIC}$ : 13.3). However, only 3 out of 24 individuals were allocated to the second group, and they also had some uncertainty to their classification while all individuals in the primary group were perfectly assigned. Therefore, based on stable isotope ratios and the low variability in stomach contents, all DR whitefish individuals seemed to belong to one group of planktivorous specialized fish. Within LSR whitefish two ecological clusters were identified with moderate to very strong support within all three lakes ( $\triangle \mathrm{BIC}$ in LP: 3.8, SU: 4.5, VG: 13.5). The assignment of individuals to the two groups correlated directly with littoral and profundal habitats in which the individual had been caught (Fig. 4; correctly classified to habitat: LP: $100 \%$, SU: $92 \%$, VG: $79 \%$ ). Thus fish recorded in profundal and littoral habitats occupied two separate isotopic niches, utilizing profundal and littoral foraging resources respectively. Based on this smaller sample of fish analyzed for stable isotope ratios, we hereafter use habitat (littoral and profundal) as a proxy for the two ecological sub-groups within the LSR whitefish to increase sample sizes.

In the analysis of genetic variation at 16 microsatellite loci, none of the three ecologically different groups (i.e. DR, littoral LSR, and profundal LSR whitefish) showed departures from HWE or LE after correction for multiple tests (but see "Littoral and

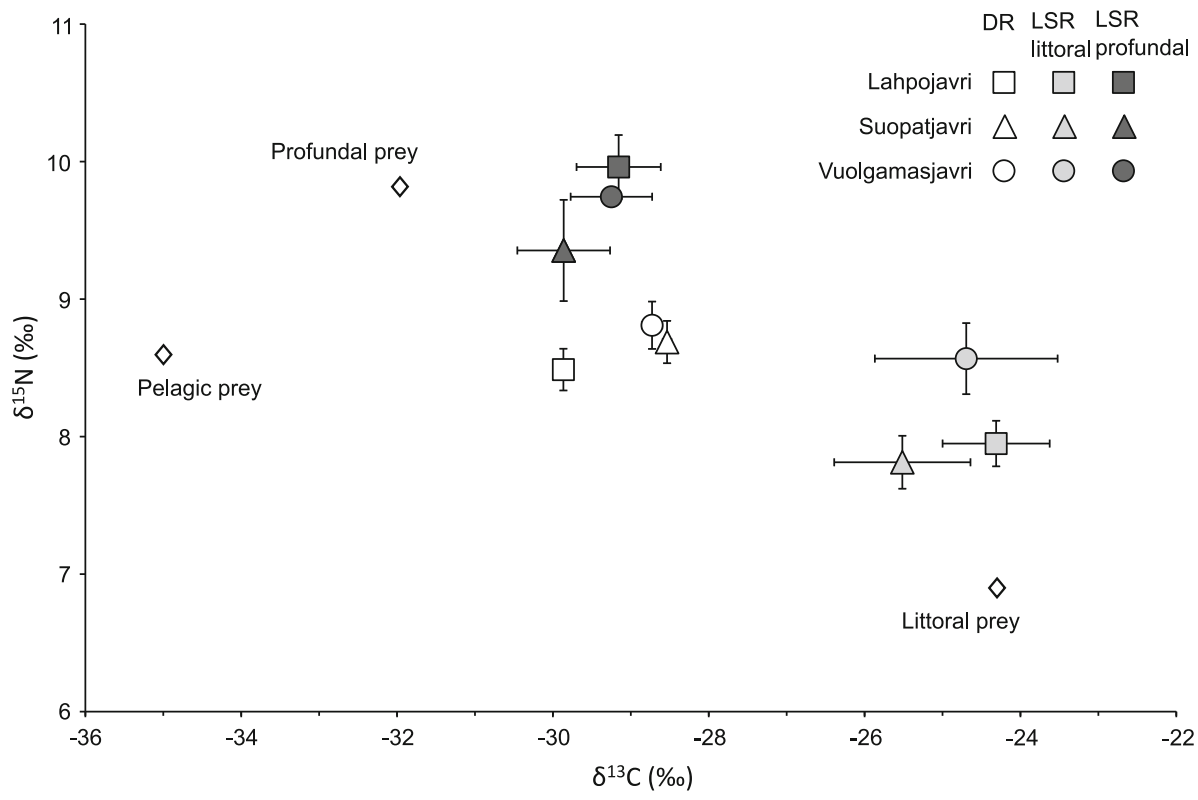

Fig. 3 Isotopic niches of densely rakered (DR) and large sparsely rakered (LSR) whitefish collected from the littoral and the profundal zone (mean $\pm 95 \%$ confidence interval). Fractionation corrected mean values of prey (diamonds) from pelagic (zooplankton), littoral (benthic macroinvertebrates), and profundal (benthic macroinvertebrates) habitats are shown for reference 


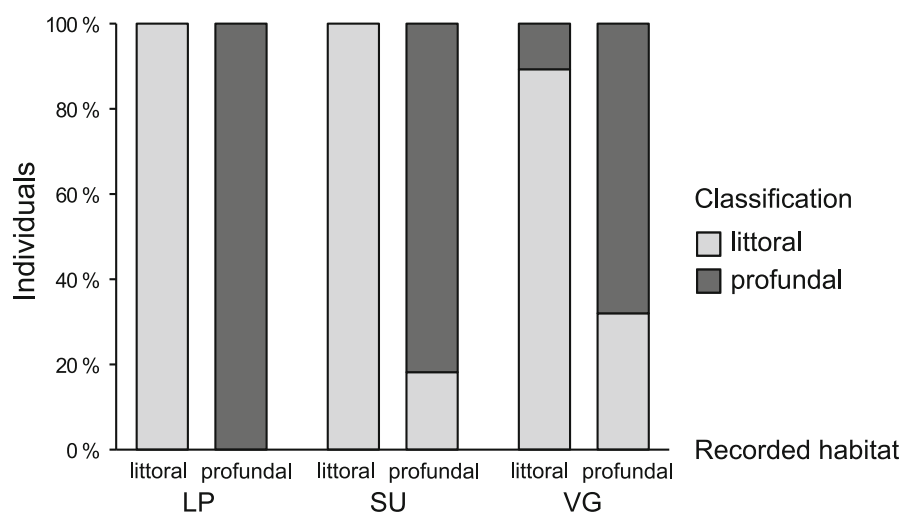

Fig. 4 Comparison of recorded habitat and individual assignment to the two ecological sub-groups within LSR whitefish in Lahpojavri (LP, $n=46$ ), Suopatjavri (SU, $n=48)$, and Vuolgamasjavri (VG, $n=53$ ). The classification was based on the results from model-based clustering using individual stable isotope ratios

profundal foraging specialisms" below). Significant genetic differences were found between the DR and the LSR whitefish collected from the littoral zone within each lake $\left(\mathrm{LP}: \mathrm{F}_{\mathrm{ST}}=0.042, \mathrm{SU}: \mathrm{F}_{\mathrm{ST}}=0.052, \mathrm{VG}: \mathrm{F}_{\mathrm{ST}}=0.096\right.$, all p-values $\left.<0.001\right)$.

Littoral and profundal foraging specialisms

Littoral and profundal caught LSR whitefish were not significantly different in body length ( $t$ test: LP: $\mathrm{F}_{1,81}=0.001, p=0.97, \mathrm{SU}: \mathrm{F}_{1,69}=0.29, p=0.59, \mathrm{VG}: \mathrm{F}_{1,109}=1.92$, $p=0.17$ ), thus excluding ontogenetic niche shifts as a cause for the observed differences. Furthermore, there was no significant difference in sex ratio between the two sub-groups in any of the lakes (Fisher's exact test: LP: $p=0.09$, SU: $p=0.45$, VG: $p=0.57$ ). However, profundal LSR whitefish had significantly lower number of gill rakers compared to littoral LSR whitefish in all three lakes (Fig. 5; $t$ test: LP: $\mathrm{F}_{1,64}=7.91, p<0.01$, SU: $\left.\mathrm{F}_{1,74}=11.66, p<0.01, \mathrm{VG} \mathrm{F}_{1,92}=17.21, p<0.001\right)$. Gill raker modality within the LSR morph was also evaluated using model-based clustering (mclust). In Lahpojavri unimodal and bimodal gill raker distributions gained equivalent support based on the low $\triangle \mathrm{BIC}$ value of 1.14. In Suopatjavri there was moderate support for bimodal gill raker distribution within the LSR morph $(\triangle \mathrm{BIC}=5.0)$. In Vuolgamasjavri bi- and trimodal gill raker distributions within the LSR morph were equally supported $(\triangle \mathrm{BIC}=0.93)$. Altogether bimodal gill raker distributions within the LSR morph were likely in all three lakes.

The fish identified a priori as LSR whitefish on the basis of external morphology but which were collected from the profundal were enriched in $\delta^{15} \mathrm{~N}$ and depleted in $\delta^{13} \mathrm{C}$ compared to those collected from the littoral in all three lakes (Fig. 3; permutation tests of centroid location, all lakes: $p=0.001$, Hotelling's $\mathrm{T}^{2}: p<0.001$ ). This difference in foraging niche utilization was confirmed by small short-term diet overlap between the two sub-groups (Shoeners index mean $=0.23$, Table S8). The sub-groups also had similar niche use across lakes, as indicated by relatively high diet similarity indices (mean Schoeners index within sub-groups between lakes: littoral LSR: 0.64, profundal LSR: 0.53 , Table S8). Stomach contents showed that diet of profundal caught LSR whitefish consisted mainly of chironomid larvae and Pisidium spp. whilst LSR whitefish 


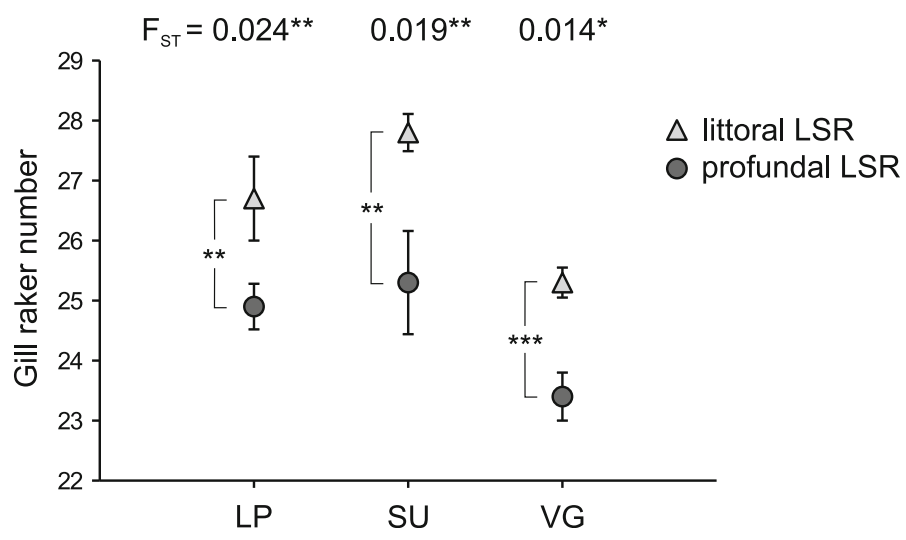

Fig. 5 Gill raker number (mean $\pm \mathrm{SE}$ ) in LSR whitefish from littoral and profundal habitats were significantly different in Lahpojavri (LP), Suopatjavri (SU), and Vuolgamasjavri (VG). Genetic differences between littoral and profundal LSR whitefish are indicated by $\mathrm{F}_{\mathrm{ST}}$-values. Significance levels are illustrated by stars, $* p<0.05, * * p<0.01, * * * p<0.001$

collected from the littoral fed on more varied prey, dominated by zooplankton, Eurycercus lamellatus, insect larvae, and snails (Fig. 2). The diet niche width was larger for the littoral (mean $\mathrm{B}=3.58$ ) compared to the profundal LSR whitefish (mean B = 2.35), and the littoral LSR whitefish also had higher levels of inter-individual diet variation (littoral: mean $\mathrm{E}=0.70$, profundal: $\mathrm{E}=0.46$ ) (Table S9). However, the diet variation was not organized into clusters in either sub-group (Table S9). Based on stable isotopes, niche width (SEA) was larger (2.2 times) in littoral LSR compared to profundal LSR whitefish in only one lake (VG: $p=0.002$ ). In this lake the littoral LSR whitefish was also the most variable in niche use (CD permutation test: $p=0.002)$. In the other two lakes the isotopic niche width was similar in the two sub-groups (LP: $p=0.36$, SU: $p=0.27$ ), and there was no significant difference in variability between them (CD permutation test LP: $p=0.07, \mathrm{SU}: p=0.16$ ) (Table S9).

Departures from the Hardy-Weinberg equilibrium were observed in profundal caught LSR from Suopatjavri at two loci, and at one locus in profundal caught LSR from Lahpojavri and in littoral caught LSR from Vuolgamasjavri (Table S3). Three out of these four cases were associated with heterozygote deficit. However, none were significant after sequential Bonferroni corrections (SBC). In the test for linkage disequilibrium two to eight out of 120 loci pairs in each of the six groups (littoral and profundal LSR within the three lakes) displayed possible linkage, with an overall across group departure of four out of 119 loci-pairs. None of the departures were significant after SBC's. When we pooled littoral and profundal caught LSR whitefish within each lake to reveal signatures of Wahlund effect, only one locus (which were non-significant after SBC) and no groups showed significant departure from HWE. However, the number of departures from LE increased to 3-12 loci pairs per combined group (compared with 2-8 in separate groups), and 11 loci pairs overall (compared to 4 in separate groups) indicative of substructure within the tested samples. Analyses of genetic variation also showed significant but small genetic differences between LSR whitefish caught in the littoral and profundal habitats within all three lakes $\left(\mathrm{LP}, \mathrm{F}_{\mathrm{ST}}=0.024, p=0.0097 ; \mathrm{SU}, \mathrm{F}_{\mathrm{ST}}=0.019, p=0.0076 ; \mathrm{VG}, \mathrm{F}_{\mathrm{ST}}=0.014\right.$, $p=0.0297)$. The STRUCTURE analysis revealed $K=1$ for Lahpojavri and Suopatjavri $($ mean $\ln \operatorname{Pr}(X \mid K)=-932 \pm 0.25(\mathrm{LP}),-1135 \pm 0.30(\mathrm{SU}))$, and $\mathrm{K}=2$ for Vuolga- 
masjavri (mean $\ln \operatorname{Pr}(X \mid K)=-1111 \pm 1.60(\mathrm{VG})$ ) (Table S10). However, the values of $\ln \operatorname{Pr}(X \mid K)$ were in general very similar with high standard deviations for all situations of $\mathrm{K}(1-3)$ within the three lakes and the determined $\mathrm{K}$ should therefore be interpreted with caution (Pritchard et al. 2000).

\section{Discussion}

Here we describe a subtle divergence in the trophic niche within a well recognized trophic polymorphism in whitefish. We show that the large sparsely rakered (LSR) whitefish morph, exhibits individuals specializing on either shallow littoral or deep profundal benthic resources. This divergence in resource use was accompanied by small but significant differences in trophic morphology (gill raker number). Genetic analyses indicated weak reproductive barriers between the two ecological sub-groups. In addition, our results confirmed the expected more profound ecological, morphological and genetic divergence between sympatric benthic LSR and pelagic densely rakered (DR) whitefish morphs (e.g. Østbye et al. 2006; Harrod et al. 2010). The clear differences between the two morphs (DR and LSR), and the divergence within the LSR morph between littoral and profundal resources were paralleled in all three lakes included in this study. These parallel patterns of specializations suggest that similar natural selection pressures are acting in all three lakes, maintaining and/or driving the divergence (Endler 1986; Schluter 2000).

We observed a clearly defined and consistent divergence into pelagic and benthic specialists indicated by low overlap in resource use (habitat and diet), and divergent gill raker number ranges between the DR and LSR whitefish morphs. Similar pelagic and benthic specialists are also well documented in whitefish from other lakes (Amundsen et al. 2004b; Kahilainen et al. 2004; Østbye et al. 2006), and in other fish species (reviewed in Schluter and McPhail 1993; Robinson and Wilson 1994). The DR morph was highly specialized on pelagic food resources, as indicated by narrow resource niche use, low interindividual diet variation, and strong association with the pelagic habitat in all three lakes. This pelagic specialization was also reflected morphologically, in the relatively high number of gill rakers, an adaptation to planktivory (Kahilainen et al. 2011). In some cases pelagic-benthic specialization in fish has led to reproductively isolated species pairs (e.g. Lu et al. 2001; McKinnon and Rundle 2002). In the present study, analyses of genetic divergence showed that sympatric DR and LSR whitefish morphs were reproductively isolated. The $\mathrm{F}_{\mathrm{ST}^{-}}$-values in this study were similar to previous studies of these whitefish morphs from nine different lakes, which reported values ranging from 0.010-0.075 (Østbye et al. 2006; Præbel et al. in press). Reproductive isolation seems to be the general situation between morphologically and ecologically distinct DR and LSR whitefish morphs in northern Fennoscandia, and is likely related to differences in spawning times and places (Østbye et al. 2005b; Vonlanthen et al. 2009).

Ecological opportunity is generally considered to be a prerequisite for evolutionary diversification, and limit the number of new types formed in a radiation process (Losos and Schluter 2000; Schluter 2000; Seehausen 2006). However, even with ecological opportunity present, species may fail to diversify for a number of reasons, e.g. genetic constraints and time for divergence (Taylor and McPhail 2000; Coyne and Orr 2004; Barrett and Schluter 2008). Constraints related to the colonization history, in addition to lower levels of ecological opportunity, have been used to explain the absence of specialist profundal whitefish morphs (small sparsely rakered, SSR) in the Alta-Kautokeino watercourse (Siwertsson et al. 2010). The three lakes included in the present study all had well 
developed pelagic, littoral, and profundal zones, but no individuals of the SSR morph were identified based on the a priori morph classification. However, within the LSR morph two clear and discrete ecological sub-groups were identified based on stable isotope ratios, specializing on littoral and profundal foraging resources, respectively. The sub-groups identified by long-term resource utilization (stable isotopes) mapped closely on to habitat use, and the correspondence of the diet based on stomach contents with the results of stable isotopes indicates that the observed diet differences were sustained over long time periods (at least months). Thus, these fish did not seem to migrate extensively between the different benthic habitats (littoral and profundal), at least not for feeding purposes. The ecological sub-division of the LSR morph could not be explained by ontogenetic niche shifts or differences between sexes, which are two main sources of niche variation within populations (Bolnick et al. 2003). However, subtle but significant differences in number of gill rakers were observed, possibly constituting morphological adaptations to the different foraging niches. Profundal habitats in postglacial lakes are very different from littoral habitats (Klemetsen 2010) and require special adaptations in foraging behavior and morphology (Klemetsen et al. 2002; Kahilainen and Østbye 2006; Harrod et al. 2010). Such adaptations to a profundal niche were shown to be heritable in experiments with Arctic charr (Klemetsen et al. 2002, 2006). Here we also found significant genetic differences between LSR whitefish from littoral and profundal habitats. The divergence was supported by signatures of Wahlund effect in the pooled samples of individuals from the two habitats. The genetic differences were however small, which was also evident from the Bayesian clustering in STRUCTURE failing to clearly identify more than one genetic group. This may, in part, be due to lack of departures from HWE and LE as identified in the exact tests and the general ability of STRUCTURE to cluster individuals in situations with low genetic differentiation (see e.g. Schwartz and McKelvey 2008; Kalinowski 2011). Taken together, consistent weak but significant genetic differentiation was identified between the littoral and profundal whitefish specialists. Whether the weak differentiation is due to weaker divergent natural selection (Nosil et al. 2009) or a more recent divergence (Coyne and Orr 2004) compared to the DR and LSR whitefish divergence remains to be explored.

Natural populations often vary in their degree of ecological and morphological divergence and completeness of reproductive isolation. This variation can be arranged along an ecological speciation continuum of increasingly discrete divergence, from small-scale inter-individual variation in panmictic populations, to ecotypes and discrete polymorphisms within species, and finally to completely reproductively isolated species (Smith and Skúlason 1996; Hendry 2009; Nosil et al. 2009; Seehausen 2009). The discrete DR and LSR whitefish morphs are a good example of a clear polymorphism. They are clearly separated in niche use, morphological traits and are also reproductively isolated (e.g. Amundsen et al. 2004b; Kahilainen and Østbye 2006; Østbye et al. 2006; this study). A more subtle divergence was documented between littoral and profundal specialists within the LSR morph. They showed profound ecological differences, but were less morphologically and genetically divergent. Similar subtle splits have recently been identified also within one of two sympatric Arctic charr morphs (Knudsen et al. 2010, 2011). The two levels of divergence (between morphs and within the LSR morph) observed here may thus represent different stages in the process of ecological speciation.

Behavioral specializations, such as diet choice, are generally plastic and reversible, and expected to precede morphological adaptations in various radiation and speciation models (West-Eberhard 1989; Wimberger 1994; Skúlason and Smith 1995; Price et al. 2003; Streelman and Danley 2003). The differences in diet and isotope values (reflecting foraging behavior) were about the same magnitude between the littoral and profundal specializing 
LSR whitefish in this study as between the LSR and SSR morphs in lakes where three sympatric whitefish morphs co-occur (Kahilainen et al. 2003; Harrod et al. 2010). This indicates that the divergence we observe within the LSR morph in this study is ecologically as profound as between separate morphs in other lakes. Inter-individual variation in trophic ecology within populations is an important starting point for further divergence and the evolution of polymorphisms and ultimately species (Bolnick et al. 2003). However, differences in gill raker number and genetic divergence were less developed between the specialist groups of LSR in the present study (Kahilainen et al. 2003; Harrod et al. 2010; Præbel et al. in press). Thus, these lakes with apparently only two morphologically distinct morphs may be in the process of evolution towards three specialist morphs. The mechanisms initiating the divergence within the LSR morph are not evident but most likely involve divergent natural selection generated by frequency-dependent ecological interactions and resource acquisition. Progress along the speciation continuum towards more differentiated populations is however not inevitable, and some models and data indicate that populations may remain in one stage or even collapse to a hybrid swarm (Bolnick and Fitzpatrick 2007; Seehausen et al. 2008a; Hendry et al. 2009; Bolnick 2011; Vonlanthen et al. 2012).

Genetic analyses have suggested that within-lake postglacial divergence is the most likely origin of the DR and LSR morphs in northern Fennoscandia (Østbye et al. 2005a, 2006), thus supporting a parallel ecological speciation scenario. The LSR whitefish morph is the most generalized morph with wide ecological niches in lakes with both two and three morphs (Harrod et al. 2010; this study), and also the only morph known to occur in allopatry in northern Fennoscandia. Østbye et al. (2006) suggested a possible scenario in which the DR morph diverged from a generalist LSR population first in resource use and later also showing morphological adaptations to the pelagic niche. Adaptations to different benthic foraging niches (i.e. littoral and profundal) within the LSR morph may have evolved in a similar process observed as ecological differences in the present study and more profound morphological adaptations in lakes with three sympatric whitefish morphs (e.g. Harrod et al. 2010). Thus, two specialist morphs, to pelagic and profundal resources, respectively, may have evolved from a generalist ancestral population of LSR whitefish.

In conclusion, a common pattern of resource specialism to pelagic, littoral and profundal resources was observed in the three whitefish study systems. We found profound ecological, morphological and genetic differences between the well recognized DR and LSR morphs, in addition to divergence between different benthic foraging niches (littoral and profundal) within the LSR whitefish morphs. These two levels of divergence may represent different stages in the process of ecological speciation. The results were paralleled in three different lakes, suggesting divergent natural selection being the force driving and/or maintaining the observed differences. The ecological differences within the LSR morph were of the same magnitude as differences observed between specialized littoral and profundal whitefish morphs in other lakes, which suggest that these populations may be in the process of divergence towards separate morphs. As natural scenarios of ongoing splitting, these are promising systems for future empirical speciation research, in particular in relation to studies of the incipient phase of the divergence process.

Acknowledgments We thank L. Dalsbø, T. Hanebrekke, C. Lien and other members of the Freshwater Ecology Group and the Fish Biology and Population Genetic Group at the University of Troms $\varnothing$ for field and technical assistance. This research was funded by the Norwegian Research Council (NFR 186320/V40). Stable isotope analyses were funded by the NERC LSMSF steering committee (EK-118-01/08). 
Open Access This article is distributed under the terms of the Creative Commons Attribution License which permits any use, distribution, and reproduction in any medium, provided the original author(s) and the source are credited.

\section{References}

Adams CE, Fraser D, Huntingford FA, Greer RB, Askew CM, Walker AF (1998) Trophic polymorphism amongst Arctic charr from Loch Rannoch, Scotland. J Fish Biol 52:1259-1271

Amundsen P-A (1988) Habitat and food segregation of two sympatric populations of whitefish (Coregonus lavaretus L. s.l.) in Stuorajavri, northern Norway. Nord J Freshwater Res 64:67-73

Amundsen P-A (1995) Feeding strategy of Arctic charr (Salvelinus alpinus): general opportunist but individual specialist. Nord J Freshwater Res 71:150-156

Amundsen P-A, Bøhn T, Våga GH (2004a) Gill raker morphology and feeding ecology of two sympatric morphs of European whitefish (Coregonus lavaretus). Ann Zool Fenn 41:291-300

Amundsen P-A, Knudsen R, Klemetsen A, Kristoffersen R (2004b) Resource competition and interactive segregation between sympatric whitefish morphs. Ann Zool Fenn 41:301-307

Araujo MS, Guimaraes PR, Svanbäck R, Pinheiro A, Guimaraes P, Dos Reis SF, Bolnick DI (2008) Network analysis reveals contrasting effects of intraspecific competition on individual vs. population diets. Ecology 89:1981-1993

Barrett RDH, Schluter D (2008) Adaptation from standing genetic variation. Trends Ecol Evol 23:38-44

Bolnick DI (2011) Sympatric speciation in threespine stickleback: why not? Int J Ecol 2011:1-15

Bolnick DI, Fitzpatrick BM (2007) Sympatric speciation: models and empirical evidence. Annu Rev Ecol Evol Syst 38:459-487

Bolnick DI, Yang LH, Fordyce JA, Davis JM, Svanbäck R (2002) Measuring individual-level resource specialization. Ecology 83:2936-2941

Bolnick DI, Svanbäck R, Fordyce JA, Yang LH, Davis JM, Hulsey CD, Forister ML (2003) The ecology of individuals: incidence and implications of individual specialization. Am Nat 161:1-28

Coyne JA, Orr HA (2004) Speciation. Sinauer Associates, Sunderland

Earl DA, vonHoldt BM (2011) STRUCTURE HARVESTER: a website and program for visualizing STRUCTURE output and implementing the Evanno method. Conservation Genet Resour

Endler JA (1986) Natural selection in the wild. Princeton University Press, Princeton

Excoffier L, Lischer HEL (2010) Arlequin suite ver 3.5: a new series of programs to perform population genetics analyses under Linux and Windows. Mol Ecol Resour 10:564-567

Fraley C, Raftery AE (2002) Model-based clustering, discriminant analysis, and density estimation. JASA 97:611-631

Fraley C, Raftery AE (2006) MCLUST version 3 for R: Normal mixture modeling and model-based clustering. Technical report no. 504. Department of statistics, University of Washington. Available at: http://www.stat.washington.edu/research/reports/2006/tr504.pdf

Guo SW, Thompson EA (1992) Performing the exact test of Hardy-Weinberg proportion for multiple alleles. Biometrics 48:361-372

Harrod C, Mallela J, Kahilainen KK (2010) Phenotype-environment correlations in a putative whitefish adaptive radiation. J Anim Ecol 79:1057-1068

Hendry AP (2009) Ecological speciation! Or the lack thereof? Can J Fish Aquat Sci 66:1383-1398

Hendry AP, Bolnick DI, Berner D, Peichel CL (2009) Along the speciation continuum in sticklebacks. J Fish Biol 75:2000-2036

Herder F, Schliewen UK (2010) Beyond sympatric speciation: radiation in sailfin silverside fishes in the Malili Lakes (Sulawesi). In: Glaubrecht M (ed) Evolution in action: case studies in adaptive radiation, speciation and the origin of biodiversity. Springer, Heidelberg, pp 465-483

Hubisz MJ, Falush D, Stephens M, Pritchard JK (2009) Inferring weak population structure with the assistance of sample group information. Mol Ecol Resour 9:1322-1332

Jackson AL, Inger R, Parnell AC, Bearhop S (2011) Comparing isotopic niche widths among and within communities: SIBER-Stable Isotope Bayesian Ellipses in R. J Anim Ecol 80:595-602

Jacob U, Mintenbeck K, Brey T, Knust R, Beyer K (2005) Stable isotope food web studies: a case for standardized sample treatment. Mar Ecol Prog Ser 287:251-253

Kahilainen K, Østbye K (2006) Morphological differentiation and resource polymorphism in three sympatric whitefish Coregonus lavaretus (L.) forms in a subarctic lake. J Fish Biol 68:63-79 
Kahilainen K, Lehtonen H, Könönen K (2003) Consequence of habitat segregation to growth rate of two sparsely rakered whitefish (Coregonus lavaretus (L.)) forms in a subarctic lake. Ecol Freshw Fish 12:275-285

Kahilainen K, Malinen T, Tuomaala A, Lehtonen H (2004) Diel and seasonal habitat and food segregation of three sympatric Coregonus lavaretus forms in a subarctic lake. J Fish Biol 64:418-434

Kahilainen KK, Siwertsson A, Gjelland KØ, Knudsen R, Bøhn T, Amundsen P-A (2011) The role of gill raker number variability in adaptive radiation of coregonid fish. Evol Ecol 25:573-588

Kalinowski ST (2011) The computer program STRUCTURE does not reliably identify the main genetic clusters within species: simulations and implications for human population structure. Heredity 106:625-632

Kang C-K, Kim JB, Lee K-S, Kim JB, Lee P-Y, Hong J-S (2003) Trophic importance of benthic microalgae to macrozoobenthos in coastal bay systems in Korea: dual stable $\mathrm{C}$ and $\mathrm{N}$ isotope analyses. Mar Ecol Prog Ser 259:79-92

Kass RE, Raftery AE (1995) Bayes factors. JASA 90:773-795

Kiljunen M, Grey J, Sinisalo T, Harrod C, Immonen H, Jones RI (2006) A revised model for lipidnormalizing $\delta^{13} \mathrm{C}$ values from aquatic organisms, with implications for isotope mixing models. $\mathrm{J}$ Appl Ecol 43:1213-1222

Klemetsen A (2010) The charr problem revisited: exceptional phenotypic plasticity promotes ecological speciation in postglacial lakes. Freshw Rev 3:49-74

Klemetsen A, Elliott JM, Knudsen R, Sørensen P (2002) Evidence for genetic differences in the offspring of two sympatric morphs of Arctic charr. J Fish Biol 60:933-950

Klemetsen A, Knudsen R, Primicerio R, Amundsen P-A (2006) Divergent, genetically based feeding behaviour of two sympatric Arctic charr, Salvelinus alpinus (L.), morphs. Ecol Freshw Fish 15:350-355

Knudsen R, Amundsen P-A, Klemetsen A (2003) Inter- and intra-morph patterns in helminth communities of sympatric whitefish morphs. J Fish Biol 62:847-859

Knudsen R, Klemetsen A, Amundsen P-A, Hermansen B (2006) Incipient speciation through niche expansion: an example from the Arctic charr in a subarctic lake. Proc R Soc B 273:2291-2298

Knudsen R, Primicerio R, Amundsen P-A, Klemetsen A (2010) Temporal stability of individual feeding specialization may promote speciation. J Anim Ecol 79:161-168

Knudsen R, Siwertsson A, Adams CE, Garduño-Paz M, Newton J, Amundsen PA (2011) Temporal stability of niche use exposes sympatric Arctic charr to alternative selection pressures. Evol Ecol 25:589-604

Kocher TD (2004) Adaptive evolution and explosive speciation: the cichlid fish model. Nat Rev Genet 5:288-298

Kristjánsson BK, Skúlason S, Noakes DLG (2002) Morphological segregation of Icelandic threespine stickleback (Gasterosteus aculeatus L). Biol J Linn Soc 76:247-257

Layman CA, Arrington DA, Montana CG, Post DM (2007a) Can stable isotope ratios provide for community-wide measures of trophic structure? Ecology 88:42-48

Layman CA, Quattrochi JP, Peyer CM, Allgeier JE (2007b) Niche width collapse in a resilient top predator following ecosystem fragmentation. Ecol Lett 10:937-944

Levins R (1968) Evolution in changing environments: some theoretical explorations. Princeton University Press, Princeton

Losos JB, Schluter D (2000) Analysis of an evolutionary species-area relationship. Nature 408:847-850

Lu G, Basley DJ, Bernatchez L (2001) Contrasting patterns of mitochondrial DNA and microsatellite introgressive hybridization between lineages of lake whitefish (Coregonus clupeaformis); relevance for speciation. Mol Ecol 10:965-985

Malmquist HJ, Snorrason SS, Skúlason S, Jonsson B, Sandlund OT, Jónasson PM (1992) Diet differentiation in polymorphic Arctic charr in Thingvallavatn, Iceland. J Anim Ecol 61:21-35

McKinnon JS, Rundle HD (2002) Speciation in nature: the threespine stickleback model systems. Trends Ecol Evol 17:480-488

Newsome SD, del Rio CM, Bearhop S, Phillips DL (2007) A niche for isotopic ecology. Front Ecol Environ 5:429-436

Nosil P (2012) Ecological speciation. Oxford University Press, Oxford

Nosil P, Harmon LJ, Seehausen O (2009) Ecological explanations for (incomplete) speciation. Trends Ecol Evol 24:145-156

Østbye K, Bernatchez L, Næsje TF, Himberg KJM, Hindar K (2005a) Evolutionary history of the European whitefish Coregonus lavaretus (L.) species complex as inferred from mtDNA phylogeography and gillraker numbers. Mol Ecol 14:4371-4387

Østbye K, Næsje TF, Bernatchez L, Sandlund OT, Hindar K (2005b) Morphological divergence and origin of sympatric populations of European whitefish (Coregonus lavaretus L.) in Lake Femund, Norway. J Evol Biol 18:683-702 
Østbye K, Amundsen P-A, Bernatchez L, Klemetsen A, Knudsen R, Kristoffersen R, Næsje TF, Hindar K (2006) Parallel evolution of ecomorphological traits in the European whitefish Coregonus lavaretus (L.) species complex during postglacial times. Mol Ecol 15:3983-4001

Parnell AC, Inger R, Bearhop S, Jackson AL (2010) Source partitioning using stable isotopes: coping with too much variation. PLoS ONE 5:e9672

Peccoud J, Ollivier A, Plantegenest M, Simon JC (2009) A continuum of genetic divergence from sympatric host races to species in the pea aphid complex. PNAS 106:7495-7500

Perga ME, Gerdeaux D (2005) 'Are fish what they eat' all year round? Oecologia 144:598-606

Post DM (2002) Using stable isotopes to estimate trophic position: models, methods, and assumptions. Ecology 83:703-718

Post DM, Layman CA, Arrington DA, Takimoto G, Quattrochi J, Montaña CG (2007) Getting to the fat of the matter: models, methods and assumptions for dealing with lipids in stable isotope analyses. Oecologia 152:179-189

Præbel K, Westgaard JI, Amundsen P-A, Siwertsson A, Knudsen R, Kahilainen KK, Fevolden S-E (in press) A diagnostic tool for efficient analysis of population structure, hybridization and conservation status of European whitefish (Coregonus lavaretus (L.)) and vendace (C. albula (L.)). Fundam Appl LimnolAdvanc Limnol

Price TD, Qvarnström A, Irwin DE (2003) The role of phenotypic plasticity in driving genetic evolution. Proc R Soc Lond B 270:1433-1440

Pritchard JK, Stephens M, Donnelly P (2000) Inference of population structure using multilocus genotype data. Genetics 155:945-959

Puebla O (2009) Ecological speciation in marine v. freshwater fishes. J Fish Biol 75:960-996

Quevedo M, Svanbäck R, Eklöv P (2009) Intrapopulation niche partitioning in a generalist predator limits food web connectivity. Ecology 90:2263-2274

R Development Core Team (2011) R: a language and environment for statistical computing. R Foundation for Statistical Computing, Vienna

Räsänen K, Hendry AP (2008) Disentangling interactions between adaptive divergence and gene flow when ecology drives diversification. Ecol Lett 11:624-636

Rice WR (1989) Analyzing tables of statistical tests. Evolution 43:223-225

Robinson BW, Wilson DS (1994) Character release and displacement in fishes: a neglected literature. Am Nat 144:596-627

Rousset F (2008) Genepop'007: a complete re-implementation of the Genepop software for Windows and Linux. Mol Ecol Resour 8:103-106

Rundle HD, Nosil P (2005) Ecological speciation. Ecol Lett 8:336-352

Sandlund OT, Næsje TF, Jonsson B (1992) Ontogenic changes in habitat use by whitefish, Coregonus lavaretus. Environ Biol Fishes 33:341-349

Schluter D (2000) The ecology of adaptive radiation. Oxford University Press, Oxford

Schluter D (2001) Ecology and the origin of species. Trends Ecol Evol 16:372-380

Schluter D, McPhail JD (1993) Character displacement and replicate adaptive radiation. Trends Ecol Evol 8:197-200

Schoener TW (1970) Nonsynchronous spatial overlap of lizards in patchy habitats. Ecology 51:408-418

Schwartz MK, McKelvey KS (2008) Why sampling scheme matters: the effect of sampling scheme on landscape genetic results. Conserv Gen 10:441-452

Seehausen O (2006) African cichlid fish: a model system in adaptive radiation research. Proc R Soc B 273:1987-1998

Seehausen O (2009) Progressive levels of trait divergence along a 'speciation transect' in Lake Victoria cichlid fish Pundamilia. In: Butlin R, Bridle J, Schluter D (eds) Speciation and patterns of diversity. Cambridge University Press, Cambridge, pp 155-176

Seehausen O, Takimoto G, Roy D, Jokela J (2008a) Speciation reversal and biodiversity dynamics with hybridization in changing environments. Mol Ecol 17:30-44

Seehausen O, Terai Y, Magalhaes IS, Carleton KL, Mrosso HDJ, Miyagi R, van der Sluijs I et al (2008b) Speciation through sensory drive in cichlid fish. Nature 455:620-627

Servedio MR, Noor MAF (2003) The role of reinforcement in speciation: theory and data. Annu Rev Ecol Evol Syst 34:339-364

Siwertsson A, Knudsen R, Kahilainen KK, Præbel K, Primicerio R, Amundsen P-A (2010) Sympatric diversification as influenced by ecological opportunity and historical contingency in a young species lineage of whitefish. Evol Ecol Res 12:929-947

Skúlason S, Smith TB (1995) Resource polymorphisms in vertebrates. Trends Ecol Evol 10:366-370 
Skúlason S, Snorrason SS, Jonsson B (1999) Sympatric morphs, populations and speciation in freshwater fish with emphasis on arctic charr. In: Magurran AE, May RM (eds) Evolution of biological diversity. Oxford University Press, Oxford, pp 70-92

Smith TB, Skúlason S (1996) Evolutionary significance of resource polymorphisms in fishes, amphibians, and birds. Annu Rev Ecol Syst 27:111-133

Snorrason SS, Skúlason S, Jonsson B, Malmquist HJ, Jónasson PM, Sandlund OT, Lindem T (1994) Trophic specialization in Arctic charr Salvelinus alpinus (Pisces; Salmonidae): morphological divergence and ontogenic niche shifts. Biol J Linn Soc 52:1-18

Streelman JT, Danley PD (2003) The stages of vertebrate evolutionary radiation. Trends Ecol Evol 18:126-131

Syväranta J, Hämäläinen H, Jones RI (2006) Within-lake variability in carbon and nitrogen stable isotope signatures. Freshwater Biol 51:1090-1102

Taylor EB, McPhail JD (2000) Historical contingency and ecological determinism interact to prime speciation in sticklebacks, Gasterosteus. Proc R Soc Lond B 267:2375-2384

Turner TF, Collyer ML, Krabbenhoft TJ (2010) A general hypothesis-testing framework for stable isotope ratios in ecological studies. Ecology 91:2227-2233

Van Oosterhout C, Hutchinson WF, Wills DPM, Shipley P (2004) MICRO-CHECKER: software for identifying and correcting genotyping errors in microsatellite data. Mol Ecol Notes 4:535-538

Vander Zanden MJ, Rasmussen JB (1999) Primary consumer $\delta^{13} \mathrm{C}$ and $\delta^{15} \mathrm{~N}$ and the trophic position of aquatic consumers. Ecology 80:1395-1404

Vonlanthen P, Roy D, Hudson AG, Largiader CR, Bittner D, Seehausen O (2009) Divergence along a steep ecological gradient in lake whitefish (Coregonus sp.). J Evol Biol 22:498-514

Vonlanthen P, Bittner D, Hudson AG, Young KA, Müller R, Lundsgaard-Hansen B, Roy D, Di Piazza S, Largiader CR, Seehausen O (2012) Eutrophication causes speciation reversal in whitefish adaptive radiations. Nature 482:357-363

Wallace RK (1981) An assessment of diet-overlap indexes. Trans Am Fish Soc 110:72-76

Weir BS, Cockerham CC (1984) Estimating F-statistics for the analysis of population-structure. Evolution 38:1358-1370

West-Eberhard MJ (1989) Phenotypic plasticity and the origins of diversity. Annu Rev Ecol Syst 20:249-278

Wimberger PH (1994) Trophic polymorphisms, plasticity, and speciation in vertebrates. In: Stouder DJ, Fresh K, Feller RJ (eds) Theory and application in fish feeding ecology. University of South Carolina Press, Columbia, pp 19-43 